\title{
Das Ärgernis \\ der Philosophie
}

\author{
Metaphysik \\ in Adornos Negativer Dialektik
}

Herausgegeben von

Marc Nicolas Sommer und Mario Schärli

Mohr Siebeck 
Marc Nicolas Sommer ist wissenschaftlicher Assistent an der Professur für Geschichte der Philosophie an der Universität Basel.

Mario Schärli ist Diplomassistent im Fachbereich neuzeitliche und zeitgenössische Philosophie an der Universität Freiburg i.Ü.

Gedruckt mit Unterstützung der Berta Hess-Cohn Stiftung, Basel.

ISBN 978-3-16-156652-3 / eISBN 978-3-16-156653-0

DOI 10.1628/978-3-16-156653-0

ISSN 2191-6683 / eISSN 2568-6615 (Collegium Metaphysicum)

Die Deutsche Nationalbibliothek verzeichnet diese Publikation in der Deutschen Nationalbibliographie; detaillierte bibliographische Daten sind über $h t t p: / / d n b . d n b . d e$ abrufbar.

\section{(c) 2019 Mohr Siebeck Tübingen. www.mohrsiebeck.com}

Das Werk einschließlich aller seiner Teile ist urheberrechtlich geschützt. Jede Verwertung außerhalb der engen Grenzen des Urheberrechtsgesetzes ist ohne Zustimmung des Verlags unzulässig und strafbar. Das gilt insbesondere für die Verbreitung, Vervielfältigung, Übersetzung und die Einspeicherung und Verarbeitung in elektronischen Systemen.

Das Buch wurde von Computersatz Staiger in Rottenburg/N. aus der Minion gesetzt, von Gulde Druck in Tübingen auf alterungsbeständiges Werkdruckpapier gedruckt und von der Buchbinderei Spinner in Ottersweier gebunden.

Printed in Germany. 


\section{Inhaltsverzeichnis}

Hinweise zur Zitierweise $\ldots \ldots \ldots \ldots \ldots \ldots \ldots \ldots \ldots \ldots \ldots \ldots$, VII

Danksagung $\ldots \ldots \ldots \ldots \ldots \ldots \ldots \ldots \ldots \ldots \ldots \ldots \ldots \ldots \ldots \ldots \ldots \ldots \ldots$, IX

Marc Nicolas Sommer

Einleitung - Das Ärgernis der Philosophie .................... 1

\section{Begriffskritik}

Guido Kreis

,Nichtidentität' als Reflexionskategorie.

Zum systematischen Zentrum der Negativen Dialektik ........... 13

Christian Iber

Kritik jeglicher begrifflicher Bestimmung einer Sache. Wie geht das?

Zur Erkenntniskritik bei Adorno, Lyotard und Derrida .............

Tilo Wesche

Erkenntnis- und Gesellschaftskritik bei Adorno ............... $\quad 65$

Philip Hogh

Sprachformen. Zum Verhältnis von Philosophie, Rhetorik, Alltags- und Wissenschaftssprache

Emil Angehrn

,Zu sagen, was nicht sich sagen lässt'.

Zwischen Transzendenz und Erinnerung 


\section{Materialismus}

Gunnar Hindrichs

Adornos kritischer Materialismus

Dirk Braunstein, Julia Jopp und Ansgar Martins

Häretischer Materialismus.

Auf dem Weg zu einer Phänomenologie des Widergeistes

\section{Wahrheit und Geschichte}

\section{Brian O'Connor}

Adornos philosophische Wahrheiten und Wir .

Peggy H. Breitenstein

„Zu sagen, was der Mensch sei, ist absolut unmöglich.“

Zugänge und Überlegungen zum zweiten Modell der Negativen Dialektik

\section{Das Absolute}

Axel Hutter

Kritische Metaphysik. Adornos Radikalisierung der Kritischen Theorie .

\section{Mario Schärli}

Eine jede Philosophie dreht sich um den ontologischen Gottesbeweis?

Die Spur natürlicher Theologie bei Adorno

Marc Nicolas Sommer

Kritische Theorie als Phänomenologie des Absoluten.

Adornos Negative Dialektik

Literaturverzeichnis 


\title{
,Zu sagen, was nicht sich sagen lässt ${ }^{`}$ \\ Zwischen Transzendenz und Erinnerung
}

\author{
Emil Angehrn
}

„Gegen Wittgenstein zu sagen, was nicht sich sagen lässt" - dies ist es, was Kritikern der modernen Wissenschaft und Verstandeskultur wie Edmund Husserl

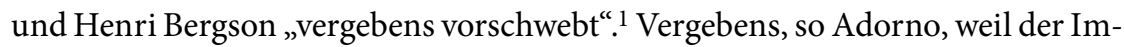
mediatismus, der beiden gemeinsam ist, nicht in der Lage ist, das Ungenügen des abstrakten Begriffs zu überwinden, über welches nur ein dialektisches Denken hinauskommt. Dass dieses jene „Utopie“ und jenes „Verlangen“ nach dem Sagen des Nicht-Sagbaren teilt, wird von Adorno mit Nachdruck unterstrichen. Die vielzitierte Passage der Negativen Dialektik, von der ich hier ausgehe, exponiert das Problem, zu einem angemessenen „Begriff von Dialektik“ zu gelangen. Deren Ziel wäre, gegenläufig zu jener Unmittelbarkeit, mit den eigenen Mittel des Begriffs das „Zurüstende und Abschneidende“ am Begriff zu ,übersteigen und dadurch ans Begriffslose" heranzureichen, oder anders formuliert: „das Begriffslose mit Begriffen aufzutun, ohne es ihnen gleichzumachen“. Die Voraussetzung dieser Option ist der an Hegel oder Platon gemahnende Glaube an die Kraft des Begriffs, „ein wie immer fragwürdiges Vertrauen darauf, dass es der Philosophie doch möglich sei“, diese paradoxe Leistung zu erbringen. Es ist ein Vertrauen, das der Philosophie nach Adornos Wort „unabdingbar" ist und ohne welches sie - „und mit ihr aller Geist" - „kapitulieren“ müsste; allein bliebe dann der nihilistische Defaitismus der Vernunft, „keine Wahrheit wäre, emphatisch wäre alles nur nichts." Soweit sind der Anspruch negativer Dialektik und eine Bedingung seiner Einlösung benannt. Wie dieser Anspruch aber einzuholen sei, worin er genau besteht, welches die Wege und Möglichkeiten seiner Realisierung sind, all dies bleibt eine offene Frage: Denn, so das nüchterne Fazit, „ein solcher Begriff von Dialektik weckt Zweifel an seiner Möglichkeit“.

Versuchen wir, die Problemkonstellation zu verdeutlichen, so haben wir als erstes zu präzisieren, worauf jene Utopie zielt, um dann zu fragen, in welcher Weise, mit welchen Mitteln philosophisches Denken ihr nahekommen, sie verwirklichen kann.

1 Theodor W. Adorno, Negative Dialektik, GS 6, 7-412, hier 21; auch die nächsten Zitate entstammen dieser Textstelle. 


\section{Das Unsagbare sagen}

Das Verlangen, das Unsagbare zu sagen, scheint sich aufzudrängen, wo immer das Denken der Endlichkeit des Erkennens und Sprechens innewird. Die zweifache Frage, ob und wieweit der Mensch in der Lage sei, die Wirklichkeit zu erkennen und sie zum Ausdruck zu bringen, beunruhigt das Denken seit der Sophistik durch die Geschichte der Philosophie hindurch. Dabei spielt von vornherein eine Dichotomie in die Frage hinein, sofern die Grenze des Wissens und Sagens sowohl das Diesseits wie das Jenseits des Logos, das Vorsprachliche wie das Übersprachliche betreffen kann. Wenn zwar die Rede von einem Unaussprechlichen meist mit der Idee eines Höheren, Transzendierenden verbunden ist, so ist sie nicht darauf beschränkt; polemisch gegen eine solche Auffassung hat Hegel das „Unsagbare“, zusammen mit Gefühl und Empfindung, gerade als das „Unbedeutendste, Unwahrste“ bezeichnet. ${ }^{2}$ Gleichwohl liegt die übliche Stoßrichtung - auch in Wittgensteins Satz und Adornos Replik - in der Grenzziehung ,nach oben'. Es ist die vom gesunden Menschenverstand mit vielen Theorien geteilte Überzeugung, dass menschliches Wissen und Sprechen nicht die letzten Gründe, die tiefste Wahrheit, das umfassende Ganze zu erreichen vermag. Unaussprechlichkeit ist dann Kennzeichen nicht der von Hegel monierten Unterbestimmtheit, sondern einer positiven Transzendierung der Sprache, der auch eine ontologische Transzendenz entspricht. Die neuplatonische Tradition hat diesen Fluchtpunkt in dezidiertester Weise ins Auge gefasst, indem sie das Unsagbar-Unerkennbare geradezu als, Überseiendes' (Plotin) bestimmt. Andere Konzepte haben diese Transzendenz mit einer Selbsttranszendierung der Sprache in Verbindung gebracht, mit dem mystischen Schweigen, der indirekten Aussageform einer Negativen Theologie, oder auch mit nichtdiskursiven Formen der poetischen Rede, Ausdrucksweisen des Bildes, Potenzen der Kunst überhaupt.

Im vorliegenden Kontext sind nicht solche Figuren der primäre Adressat Adornos, wenn er das Verlangen nach dem Sagen des Nicht-Sagbaren mit ihnen teilt, doch ihm in anderer Weise nachgehen will. Wovon sich Negative Dialektik in erster Linie abhebt, ist jene andere Dialektik, die exemplarisch für den Anspruch stand, die Grenzen der gängigen, wissenschaftlich-verstandesmäßigen Erkenntnis zu transzendieren: die spekulative Dialektik Hegels. Sie ist Adorno das Nächste, weil sie, in gemeinsamer Opposition gegen die Unmittelbarkeitsphilosophie, genau jenen von Adorno beanspruchten Willen verkörpert, im Medium des Begriffs über den Begriff hinauszugehen. Das Motiv des Speers, der die Wunde heilt, die er selbst geschlagen hat, das trosas iasetai, das Adorno der Er-

2 G.W.F. Hegel, Enzyklopädie der philosophischen Wissenschaften im Grundrisse, in: Werke in zwanzig Bänden, hg. von Eva Moldenhauer/Karl Markus Michel, Frankfurt am Main 1970, Bd. 8, \$20, Anm. (= Enzyklopädie). 
kenntnis zuweist ${ }^{3}$, benennt auch ein Innerstes der Hegelschen Dialektik. Allerdings ist hier unmittelbar die von Hegel markierte und von Adorno bekräftigte Differenz zwischen einer negativen und einer positiven, in Hegels Formulierung: spekulativen Dialektik virulent. Dezidiert weist Adorno den von Hegel reklamierten Übergang vom ,Negativ-Vernünftigen` zum ,Positiv-Vernünftigen', von der ,Dialektik' zur ,Spekulation' zurück. ${ }^{4}$ Es ist eine Absage an den klassischen dialektischen Dreischritt, der nach der Entzweiung die Wiedervereinigung, die Rückkehr aus der Entäußerung statuiert. Genauer ist es eine Absage an die von der Dialektik beanspruchte Kraft des Negativen, aus sich heraus ein Positives hervorzubringen. Unter Bedingungen der generalisierten realen Negativität verharrt Kritik nach Adorno als „unbeirrte Negation“5 im Negativen, schlägt sie nicht in ein Affirmatives um. ${ }^{6}$ Allerdings ist es wichtig, auch jenes ,Dritte, das Spekulative in Hegels Sinn, nicht einfach als eine neue, höhere Positivität zu denken, welche die Schranken des endlichen Verstandes zugleich mit den Grenzen der Sprache hinter sich lässt.

Hegels Ansinnen, im Medium des Begriffs über den Begriff hinauszugelangen, hat eine exemplarische Verkörperung in der Figur des spekulativen Satzes. Mit diesem meint Hegel eine Struktur philosophischer Darstellung, die im Durchgang durch die Bewegung des Gegenstandes und die Entwicklung der Begriffsformen in der Lage ist, die analytische Differenzierung und lineare Sukzession, welche der sprachlichen Entfaltung unabdingbar ist, immanent zu überwinden und ein Ganzes zur Sprache zu bringen. Dabei interessiert nicht das Resultat des Prozesses, sondern dieser selbst: Die Wahrheit findet sich nicht in einem abschließenden, synthetisierenden Satz, sondern in der Einsicht in die Logik und Notwendigkeit der prozessualen Entfaltung, in der Reflexion des durchschrittenen Wegs. In zweifacher Weise geht solche Reflexion über die prädikative Struktur der Sprache hinaus, indem sie einerseits jenseits des Einzelsatzes die Totalität der Formbestimmungen einer Sache zur Geltung bringt, andererseits an die Stelle der Gesamtheit der Sätze die Bewegung, die ,Performanz' der begrifflichen Entfaltung setzt. Es geht beim Übergang von der Dialektik zur Spekulation nicht einfach um ein dazukommendes, höheres oder abschließendes Drittes, sondern um eine spezifische Reflexion der genuinen Prozessualität des Diskurses bzw. der ,Arbeit des Begriffs. Diese Grundhaltung wird als solche von Adorno geteilt, der daran festhält, dass dialektische Philosophie „wesentlich nicht referierbar"7, sondern nur in der konkreten Begriffsarbeit und Materialanalyse praktizierbar

\footnotetext{
3 Adorno, Negative Dialektik, 62.

${ }^{4}$ Hegel, Enzyklopädie, $\$ 81$, Zusatz 2; vgl. Theodor W. Adorno, Negative Dialektik, 27 (Fußnote).

5 Adorno, Negative Dialektik, 162.

6 Theodor W. Adorno, Metaphysik. Begriff und Probleme, hg. von Rolf Tiedemann, Frankfurt am Main 1998, 224 (= Metaphysik).

7 Adorno, Negative Dialektik, 44.
} 
ist. Die Wahrheit des Denkens liegt in dessen Bewegung und der darin sich artikulierenden Erfahrung der Sache, nicht einem davon abstrahierten, feststellbaren Resultat. Ebenso nähert sich Adorno dem Impuls des Spekulativen darin an, dass ein Element des Hinausseins über die Negativität auch in seiner Dialektik aufscheint. Die Zerrissenheit und Mangelhaftigkeit der Welt soll nicht für sich das letzte Wort sein. Sie gewinnt ihr kritisches Profil im Spiegel des Vorscheins ihres Anderen.

Dennoch will sich negative Dialektik einer bestimmten Transgression zum Höheren verweigern, die sie in Hegels System realisiert sieht. Sie widersetzt sich dem, was ihr an dieser Transgression als unhaltbar und idealisierende Hypostase, ja, als Reflex einer repressiv-destruktiven Synthese erscheint, um stattdessen am Unversöhnten festzuhalten. Die Frage ist, in welcher Weise Dialektik unter diesen Bedingungen Wittgensteins Sprachverbot widersprechen und gleichzeitig die Immanenz des Bestehenden aufsprengen kann, um dem Anderen, dem Nichtsagbaren Ausdruck zu verleihen.

Verschiedene Leitbegriffe und konzeptuelle Vorschläge kommen hier in den Blick. Sie sollen jene Defizite der prädikativen Sprache überwinden, die Hegel der Verstandeslogik, Adorno dem identifizierenden Denken anlastet. Gegen sie kommen auf der einen Seite logisch-epistemologische Konzepte zum Tragen, die jenseits des abstrakten Begriffs auf die Materialität und Besonderheit der Sache, jenseits des Verstandes auf das sinnliche und intuitive Element des Erkennens abheben. Mit ihnen verbindet sich die Absage an Ganzheit und Totalität, das Plädoyer für das Fragmentarische, die mikrologische Lektüre. Auf der Gegenseite geht es darum, in der Darstellung über das Partielle und Isolierte der Einzelaussagen hinauszugelangen und darin etwas von dem durch Hegel postulierten spekulativen Potential einzuholen. Einschlägig sind dazu die komplementären Stichworte der Konstellation und des Namens. Ausdrücklich bringt Adorno die Konstellation, die Beschreibung einer Sache im konkreten Geflecht seiner Bestimmungen, mit der Endlichkeit des Begriffs und seiner Fähigkeit zur Selbsttranszendierung zusammen: Das Ungenügen des einzelnen Begriffs nötigt dazu, „andere herbeizuzitieren; darin entspringen jene Konstellationen, an die allein von der Hoffnung des Namens etwas überging. "8 Erst die konkrete Konfiguration von Ausdrücken und Umschreibungen vermag zu realisieren, was der Begriff intendierte: „das Gemeinte ganz auszudrücken“; sie repräsentiert „von außen, was der Begriff im Inneren weggeschnitten hat “. 9 Die Hoffnung des Namens wiederum steht für jene Unmittelbarkeit der Gesamtschau, auf welche das utopische Ziel der dialektischen Erkenntnis gerichtet war. Adorno führt sie weiter aus im Zusammenhang des - namentlich von W. Benjamin besprochenen Konzepts des dialektischen Bildes, dessen Offenbarungspotential mit der Evo-

\footnotetext{
8 Adorno, Negative Dialektik, 62.

9 Adorno, Negative Dialektik, 164.
} 
kationsmacht von Namen kommuniziert, die etwas in seiner Ganzheit und Gegenwärtigkeit anwesend sein lassen.

\section{Die zweifache Nicht-Sagbarkeit}

Ich will nun nicht dieser Linie der Darstellungsformen von Name und Konstellation weiter folgen ${ }^{10}$, sondern eine andere Dimension des Ausgriffs auf das Ganze erkunden, die primär durch dessen Inhalt bestimmt wird. Und zwar ist sie durch jene Seite des Inhalts geprägt, die für die Absage an die spekulative Synthese wesentlich verantwortlich ist, die Seite der Negativität. Das Spezifische negativer Dialektik ist grundlegend durch die Negativität der Sache bestimmt, der ihr Denken und ihre Kritik gilt: die Negativität dessen, was nicht sein soll, wovor wir zurückschrecken, worunter wir leiden. Sie zur Sprache zu bringen ist die eminente, kognitive wie darstellungsmäßige Herausforderung der Philosophie. Im Blick darauf zeigt sich nun eine besondere Verstrickung mit unserer Ausgangsfrage. Die Nichtsagbarkeit des zu Sagenden, auf die wir zuerst als Nichtsagbarkeit des wahrhaft Seienden und Überseienden, des umfassenden Ganzen und höchsten Wahren gestoßen sind, kehrt in eigentümlicher Spiegelung wieder in der Nichtsagbarkeit des Nichtseins, des absoluten Leidens, des Todes.

Was ,nicht sich sagen lässt', zeigt sich in zweifacher Gestalt. Es gibt nicht allein die Nicht-Sagbarkeit des Höheren, transzendenten Wahren, sondern ebenso die des Niedrigeren, Unwahren, und dies nicht nur mit Bezug auf die eingangs vermerkte, von Hegel inkriminierte Version des seinsmäßig Minderen und Unbestimmten, sondern auch in dem zugespitzteren Sinn des praktisch-Negativen, des Nichtseinsollenden, Destruktiven und Abgewehrten. ${ }^{11}$ Wir haben gewissermaßen mit einer doppelten Nicht-Sagbarkeit ,nach oben' und ,nach unten` zu tun, mit der Nicht-Erreichbarkeit eines an sich Erstrebten und erkenntnismäßig Anvisierten auf der einen Seite, der Unaussprechlichkeit eines Perhorreszierten, Abgründigen auf der anderen Seite. Für die Mystik ist nicht nur Gott, sondern auch der Teufel unerkennbar, für die philosophische Ratio nicht nur der Sinn der Geschichte, sondern auch die Herkunft der Sünde unergründlich. In bestimmter Hinsicht korrespondiert diese zweifache Ausrichtung der Doppelung von methodischem und ontologischem Negativismus. Während der erste nur den Erkenntnisvorbehalt gegenüber einem das Wissen Transzendierenden markiert - exemplarisch in der Negativen Theologie, die von Gott nur sagt, was er nicht

10 Vgl. dazu Emil Angehrn, „Kritik und Versöhnung. Zur Konstellation Negativer Dialektik bei Adorno“, in: ders., Die Herausforderung des Negativen. Zwischen Sinnverlangen und Sinnentzug, Basel 2015 (= Die Herausforderung des Negativen), 43-73, hier 54-60.

${ }_{11}$ Zur Differenz von theoretischer und praktischer Negativität siehe Emil Angehrn, „Dispositive des Negativen. Grundzüge negativistischen Denkens“, in: ders., Die Herausforderung des Negativen, 14-42. 
ist, nicht, was er ist -, hat der ontologische Negativismus seinen Kern in einem seinsmäßig Negativen und Defizienten. Allerdings gehört es zur Pointe der ontologischen Negativität, dass sie sich auch erkenntnismäßig der positiven Bestimmung entzieht, wie das ungestalte mythische Chaos, Gegenstand der Urangst, nicht räumlich vermessbar und begrifflich fassbar ist. Auch diese zweite, negative Instanziierung des Nichtsagbaren kommt in der kritischen Theorie und ihrer Reflexion auf die Grenzen der Repräsentation profiliert zur Sprache. Bemerkenswert ist, dass wir hier nicht einfach mit einer faktischen Dichotomie in der inhaltlichen Besetzung und wertmäßigen Ausrichtung des Unsagbaren, zwischen einem positiven und einem negativen, einem transzendierenden und einem abgründigen Unsagbaren zu tun haben. Vielmehr geht es um ein Spannungsverhältnis, das in dieser polaren Doppelung für (negativ-)dialektisches Denken konstitutiv ist.

Ich möchte dieser Konstellation im Folgenden nicht allgemein, sondern in einer bestimmten Fokussierung nachgehen, die ihrerseits für das Denken Adornos wie anderer Exponenten der Kritischen Theorie von Belang ist. Die Darstellung dessen, was nicht sich darstellen lässt, wird darin mit dem Problem der Erinnerung verschränkt. Das Sagen des Negativen wird als Frage der Leidenserinnerung reflektiert, die einen Angelpunkt kritischer Geschichtstheorie bildet. Sie hat ihr Gegenstück in der komplementären Form der Erinnerung als Glückserinnerung, die ihrerseits in die Selbstvergewisserung kritischen Denkens hineinspielt. Die Figuren der Leidenserinnerung und der Glückserinnerung, die beide Grenzen der Darstellung berühren, bilden eine spezifische, aufklärungsbedürftige Doppelung in der normativen Abstützung dialektischen Denkens. ${ }^{12}$ Ich gehe im Folgenden beiden Figuren nach und versuche zu verdeutlichen, in welcher Weise sie, je für sich wie in ihrem Verhältnis, für die umrissene Problematik zentral und erhellend sind. Dazu sind neben den Schriften Adornos andere, auch außerphilosophische Texte hinzuzuziehen.

\section{Leidenserinnerung}

Dass das Negative, paradigmatisch: das Leiden, uns vor eine vergleichbare Herausforderung, ein vergleichbares Dilemma wie der Anspruch spekulativer Erkenntnis stellt, wird durch lebensweltliche Phänomene bezeugt und hat seinen Niederschlag im Theorieprogramm Adornos. Die leitende Devise, zu sagen, was nicht sich sagen lässt, findet ihr Echo im emphatischen Appell, „Leiden beredt werden zu lassen" ${ }^{13}$ Beide Mal geht es darum, etwas zu sagen, was gesagt wer-

12 Zum Folgenden vgl. Emil Angehrn, „Das Vergangene, das nie gegenwärtig war. Zwischen Leidenserinnerung und Glücksversprechen“, in: ders./Joachim Küchenhoff (Hgg.), Das unerledigte Vergangene. Konstellationen der Erinnerung, Weilerswist 2015, 175-205.

13 W. Adorno, Negative Dialektik, 29. 
den soll, was sich aber nicht problemlos, womöglich gar nicht sagen lässt. Die Schwierigkeit, dasjenige, was sich dem Wort, der Repräsentation entzieht, gleichwohl zum Ausdruck zu bringen, führt in vergleichbare Aporien, die sich im Falle der Negativität des zu Sagenden gegenüber dem Überbegrifflich-Transzendenten noch verschärfen. Eindringlich begegnet uns das Problem im Umgang mit der Geschichte des zwanzigsten Jahrhunderts. Einen prägnanten Ausdruck findet es in einem Gespräch zwischen den beiden Holocaust-Überlebenden Elie Wiesel und Jorge Semprun, deren literarisches Lebenswerk weithin dem Wachhalten der Erinnerung an das unsagbare Leiden gewidmet ist: „Se taire est interdit, parler est impossible“, lautet die bündige Feststellung von Elie Wiesel. ${ }^{14}$ Nicht schweigen zu dürfen, nicht sprechen zu können umreißt die Schwierigkeit, die es zu überwinden, das Dilemma, aus dem es sich zu befreien gilt.

Beides ist von Autoren profiliert herausgestellt worden: die Notwendigkeit und die Unmöglichkeit der Erinnerung. In unterschiedlicher Weise drängt sich das Erinnern-Müssen dem Bewusstsein auf: als ursprüngliche Pflicht, als treibende Kraft des historischen Gedächtnisses, als fundamentales Verlangen der Menschen. Die Unerledigtheit des Vergangenen, die dieses der Erinnerung entzieht, drängt gleichzeitig zu seiner Bewältigung und Bewusstwerdung. Profiliert ausgeführt findet sich der ethische Appell an das Gedenken des Leidens in den geschichtsphilosophischen Thesen Walter Benjamins. Die Leidenserinnerung an die Stelle des Gedächtnisses der Sieger zu setzen ist jene Umkehrung der traditionellen Historie, die Benjamin, in Radikalisierung der Historienkritik Nietzsches, einklagt. Dabei geht es nicht um eine bloße konzeptuelle Revision, sondern um das Wahrnehmen einer drängenden Aufgabe und einer Pflicht - gegen uns selbst wie gegenüber den Verstummten und Untergegangenen, den Opfern von Unrecht und Gewalt. Mit Nachdruck bekennt sich Benjamin zu diesem Auftrag, aber auch zu dieser Macht des Gedächtnisses - im Zeichen jener „schwachen messianischen Kraft“, die uns „mitgegeben“ ist und ,an welche die Vergangenheit Anspruch hat. "15 Die Forderung, Geschichte gegen den Strich zu bürsten, hat ihre Dringlichkeit als theoretisches wie als praktisches Postulat: Sie soll ein falsches, ideologisches Geschichtsbild auflösen, aber ebenso gegen die mit diesem Bild verschränkte falsche Wirklichkeit, die durch Repression und Zerstörung gekennzeichnete historische Realität Widerstand leisten. Gleichzeitig erwächst der Nachdruck des Gebots der eminenten Schwierigkeit der Erinnerung, da sie selbst im Banne der Entfremdung steht, welche sie zu überwinden sucht.

Dass die Vergegenwärtigung schmerzlicher und belastender Erlebnisse auf Hindernisse und Grenzen stößt, ist psychologisch wie kulturell vertraut. Ver-

14 Jorge Semprun/Elie Wiesel, Se taire est impossible, Paris 1995, 17.

15 Walter Benjamin, „Über den Begriff der Geschichte“, in: ders., Gesammelte Schriften, hg. von Rolf Tiedemann/Hermann Schweppenhäuser, Frankfurt am Main 1974 (= Gesammelte Schriften), Bd. I.2, 691-704, hier 694. 
gangenes ist der Retrospektion nicht umstandslos zugänglich ${ }^{16}$, erst recht dort nicht, wo es dem Bewusstsein aus nachvollziehbaren Gründen entzogen ist: wo es schmerzhaft, ängstigend, beschämend, gefährlich ist. Das psychoanalytische Theorem der Verdrängung nennt ein Paradigma der Verhüllung und Verzerrung, die ein Vergangenes der Repräsentation und willentlichen Reminiszenz entzieht. Hier kann das Vergangene nicht bewusst werden, weil es nicht bewusst werden soll, weil es nicht sein soll. Wenn zwar das normale Seelenleben auch das umgekehrte Verhältnis kennt, wonach wir Angenehmes leicht vergessen, während unangenehme und belastende Ereignisse in unserem Gedächtnis haften bleiben, so bedeutet dieses Haftenbleiben nicht schon die Fähigkeit zur Wiedererweckung, sondern kann es sich umgekehrt gerade mit Mechanismen der Verschließung und Verdeckung verknüpfen. Dabei ist der Ausschluss aus dem Bewusstsein von verschiedener Stringenz je nach der Tiefe des erlittenen oder begangenen Übels. Wenn er bei ,normalen' Schmerz- und Angsterlebnissen möglicherweise durch therapeutische Praktiken rückgängig gemacht werden kann, so bleibt er in anderen Fällen unüberwindbar. Traumatische Verletzungen können durch die Zerstörung der Ausdrucksfähigkeit verhärtet und perpetuiert, in ihrer Leidensqualität radikalisiert werden. Diese Verhärtung ist im Blick auf extreme Formen des Leidens in der Schreckensgeschichte des 20. Jahrhunderts vielfach zum Thema geworden.

Wenn Walter Benjamin schon von den Heimkehrern des Ersten Weltkriegs berichtet, dass sie verstummt aus dem Felde kamen, des ur-menschlichen Vermögens beraubt, Erfahrungen zu machen und sein Leben zu erzählen ${ }^{17}$, so nimmt diese Beraubung bei den von Primo Levi beschriebenen Muselmännern der Konzentrationslager eine neue, äußerste Gestalt an. ${ }^{18}$ Es ist jene extreme Form der Entmenschlichung, wo Individuen nicht nur geschunden und vernichtet, sondern gleichsam als leidende Menschen ausgelöscht und negiert werden, wo die Körper nicht einmal mehr zur Empfindung der Sinnlosigkeit und Artikulation des Leidens in der Lage sind. Potenziert wird dieser Sprachentzug noch in der gezielten Unterdrückung des Gedächtnisses, dem Fluch des Vergessenwerdens, der damnatio memoriae, die bewusst jeden Anspruch auf Sinn und Gedenken negiert und die Deportierten und Massakrierten dem endgültigen Nichts ausliefert. Es sind abgründige Potenzierungen des Sprach- und Gedächtnisverlusts, denen Autoren und Künstler nachgehen. Claude Lanzmann, dessen jahrzehntelange Arbeit an Shoah sich geradezu einem Unerinnerbaren,

16 Paul Ricœur bezeichnet die Selbst-Verständlichkeit des Vergangenen für die Retrospektion als eine „Pseudo-Evidenz“: Paul Ricœur, La mémoire, l'histoire, l'oubli, Paris 2000, 453 (= La mémoire, l'historie, l'oubli).

17 Walter Benjamin, „Der Erzähler. Betrachtungen zum Werk Nikolai Lesskows“, in: ders., Gesammelte Schriften, Bd. II.2, 438-465.

18 Primo Levi, Ist das ein Mensch?, Frankfurt am Main 1961. 
„wie außerhalb der menschlichen Dauer“ Verbannten widmet ${ }^{19}$, vertieft das faktische Versagen der Erinnerung und Darstellung zu einem eigentlichen Verbot ${ }^{20}$ - nicht unähnlich dem zwischen Feststellung und Verbot oszillierenden Diktum Adornos, nach Auschwitz ein Gedicht zu schreiben sei barbarisch - und verurteilt die Versuche, die totale Entmenschlichung und den massenhaften Tod medial darzustellen, ja, schon zu verstehen, als geradezu obszönes Unrecht an den Opfern. Die absolute Negativität ist im Medium der normalen Sprache und des gewohnten Bildes nicht einzuholen und nicht weiterzugeben: Der radikalen Nichtverstehbarkeit des Bösen korrespondiert die ebenso strikte Nichtrepräsentierbarkeit und Nichterinnerbarkeit.

Und doch bedeutet diese Verschärfung des Nichtsagenkönnens nichts anderes als eine Vertiefung des Anspruchs, der Forderung nach dem Ausdruck. In diesem Sinne versteht Primo Levi die Mission, welche die Überlebenden auf sich zu nehmen haben ${ }^{21}$, und auch Lanzmanns filmisches Oeuvre will nichts anderes als dem Nichtdarstellbaren jenseits der bildhaften Narration Ausdruck verleihen. Eine weitere Zuspitzung der unmöglichen Repräsentation thematisiert Giorgio Agamben mit Bezug auf die Mitglieder der, Sonderkommandos', jene einzigen wahren Zeugen der absoluten Zerstörung, die als Todgeweihte dazu verurteilt sind, nicht zu überleben und damit auch nicht vom eigenen Leiden und vom erlebten Schrecken Zeugnis ablegen zu können. ${ }^{22}$ Es ist ein Extrempunkt des Verstummens, der nach Agamben dem von Aristoteles bis Apel hochgehaltenen Dogma Hohn spricht, der Mensch sei von Natur aus ein sprechendes Lebewesen. Die vermeintliche Unhintergehbarkeit der Sprache wird obsolet angesichts der Negierbarkeit des Lebens. ${ }^{23}$ Und dennoch besteht auch hier das Pathos des Schreibens und Lesens eben darin, sich diesem Sprachentzug zu widersetzen und das Nichtgesagte, Nicht-Sagbare trotz allem zur Sprache zu bringen. Der eingangs zitierte Einspruch Adornos gegen Wittgensteins Verdikt - „wovon man nicht sprechen kann, darüber muss man schweigen“24 - findet ein entferntes Echo bei Jorge Semprun, der vor dem Hintergrund seines Erlebens im Konzentrationslager mit Entschiedenheit den anderen Satz aus dem Tractatus bestreitet: „Der Tod ist kein Ereignis des Lebens. Den Tod erlebt man nicht.“25 Dagegen beharrt Semprun nachdrücklich auf der Möglichkeit, im Hindurchgegangensein durch die Zone des Todes und Anteilnehmen am Sterben anderer vom Tode

19 Claude Lanzmann, La Tombe du divin plongeur, Paris 2012, $487 \mathrm{f}$.

20 „Un interdit de la représentation“: ebd., 536 f.; vgl. 503.

21 Primo Levi, Die Untergegangenen und die Geretteten, München 1990.

${ }^{22}$ Giorgio Agamben, Was von Auschwitz bleibt. Das Archiv und der Zeuge. Homo sacer III, Frankfurt am Main 2003.

${ }^{23}$ Ebd., 56.

${ }^{24}$ Ludwig Wittgenstein, Tractatus logico-philosophicus, in: ders., Werkausgabe, Frankfurt am Main 1984, Bd. 1, 7-85, hier $\$ 7$.

${ }^{25}$ Ebd., $\$ 6.4311$. 
Zeugnis ablegen zu können ${ }^{26}$ - ein Zeugnis, kraft dessen Semprun nicht zuletzt für Paul Ricœur zu einem Kronzeugen des Sprechens über den Tod wird. ${ }^{27}$

So sehen wir in der Konfrontation mit existentieller Negativität, mit Leiden, Unrecht und Tod, eine eigenartige Parallele zur Sprachaporie spekulativer Erkenntnis. Sie ließe sich in das die Philosophie seit Beginn beunruhigende Problem des Bösen und des Übels hinein erweitern. Adorno selbst stellt die Verbindung zwischen der fundamentalphilosophischen und der realhistorischen Provokation des Negativen her, indem er die Linie des Theodizeeproblems über das Erdbeben von Lissabon zur menschlichen Katastrophe Auschwitz auszieht und meint, dass durch die reale Hölle des menschlichen Bösen die Fähigkeit zur Metaphysik „gelähmt" worden sei. ${ }^{28}$ Es ist der Rückschlag einer essentiellen Geschichtlichkeit des Denkens, eines „Zeitkerns“ der Wahrheit ${ }^{29}$, wie er sich zuletzt auch umgekehrt in der fundamentalphilosophischen Relevanz der Erinnerung äußert.

Wenn negative Dialektik in der unnachgiebigen Negativität zugleich die Spiegelschrift des Anderen lesen will, so findet auch dieses Junktim nicht nur im Horizont der Spekulation statt. Es reflektiert sich in signifikanter Weise im Fokus der Erinnerung. Die Leidenserinnerung hat ihr Gegenbild in der Erinnerung des Glücks.

\section{Glücksversprechen}

„Die Erinnerung des fernsten und ältesten Glücks"30 - so umschreiben Max Horkheimer und Theodor W. Adorno eine Leitidee, an der sich die Zivilisationskritik der Dialektik der Aufklärung ausrichtet. Glückserinnerung erscheint zunächst als direkte Gegenfigur zur Leidenserinnerung, nicht nur mit Bezug auf die Qualität des Gegenstandes, sondern auf die Form der Vergegenwärtigung. Hat sich das Leiden als Hindernis des Erinnerns und Grund des Vergangenheitsentzugs gezeigt, so erscheint das Glück als Instanz der ursprünglichen Fülle und Gegenwärtigkeit, exemplarisch etwa in Episoden der von Proust beschriebenen mémoire involontaire, die mit der überwältigenden sinnlichen Präsenz wieder auftauchender vergangener Erlebnisse einhergeht. Gleichwohl ist es nicht so, dass vergangenes Glück sich umstandslos der Vergegenwärtigung öffnet. In Wahrheit begegnen wir auch hier einem Dilemma des Erkennens und Sprechens. Auch

26 Jorge Semprun, Schreiben oder Leben, Frankfurt am Main 1995, 230.

27 Paul Ricœur, Vivant jusqu'à la mort, suivi de Fragments, Préface d'Olivier Abel, Postface de Catherine Goldenstein, Paris 2007, $45 \mathrm{ff}$.

28 Adorno, Negative Dialektik, 354.

29 Adorno, Metaphysik, 72.

30 Max Horkheimer/Theodor W. Adorno, Dialektik der Aufklärung. Philosophische Fragmente, Frankfurt am Main 1969, 71. 
das Glück kann sich der unmittelbaren, direkten Erkenntnis entziehen. Dass wir nicht ohne weiteres wissen, was wir wollen, wenn wir uns nach dem Glück sehnen, ist nicht nur aus den Wünschen des Märchens, sondern auch der ältesten philosophischen Reflexion auf das Glück bekannt. Als umfassender Endzweck schießt das Glück nach Aristoteles über jede identifizierbare Zwecksetzung und Erfüllung hinaus. Der Mensch strebt zwar je schon nach dem Glück, aber kann es nicht in einer bestimmten Fixierung anvisieren und bewirken. ${ }^{31}$ Dass Glück in gängigen Beschreibungen mit der Überfülle, dem Unermesslichen assoziiert wird, steht nicht nur für seinen Reichtum, sondern auch seine Unfassbarkeit.

In noch anderer Weise manifestiert sich diese in der Erinnerung. Ursprüngliches Glück, wie es vielfach mit der Kindheitserinnerung verbunden ist, teilt den Status dessen, was Ernst Bloch im Bild der Heimat ins Auge fasste - „etwas, das

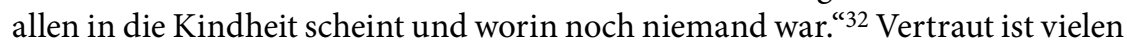
die Sehnsucht nach etwas, das sie nicht zu benennen vermögen, nach einem Ort, an dem sie noch nie waren, nach einer Erfahrung, die sie nie gemacht haben. In diesem Sinne beschreibt Augustinus die Tiefe des Gedächtnisses, das von der Suche nach etwas geleitet ist, das hinter das Erlebte und Erkannte zurückreicht, wie von der Sehnsucht „nach einem Unbekannten, das ich nie erfahren oder so völlig vergessen habe, dass ich nicht einmal mich erinnere, es vergessen $\mathrm{zu}$ haben". ${ }^{33}$ In ihrer Zeitform konvergiert diese Figur mit der eigentümlichen Idee eines „Vergangenen, das nie gegenwärtig gewesen ist“, die in herausgehobener Stellung bei Autoren wie Maurice Merleau-Ponty, Jacques Derrida und Emmanuel Lévinas wiederkehrt und für den je zurückliegenden, nie in Gegenwärtigkeit zu überführenden Ursprung steht. ${ }^{34}$ Wenn wir die Figur mit dem Topos der Kindheitserinnerung verklammern, so verbindet sich der retrospektive Entzug des Ursprungs mit dem prospektiven Offensein, mit der Erinnerung an eine Zeit der Frühe und des Anfangens, der Erwartungen und Hoffnungen. Zum Tragen kommt ein Grundzug der historischen Wahrnehmung, die im Gewesenen nicht nur das Realisierte, sondern den Hof der Virtualitäten und den offenen Prozess erkennt, nicht nur das abgeschlossene Faktum, sondern das Unerledigte erinnert. Es ist eine Erinnerung, die im Vergangenen das Unvergangene und nach vorne Treibende, die ungewordene Zukunft wahrnimmt. Wenn diese Dynamik im Horizont der Leidenserinnerung im Zeichen der Rettung, des Zurechtrückens des Entstellten stand, so steht sie im Horizont der Glückserinnerung für

31 Vgl. Emil Angehrn, „Glück und Gelingen“, in: ders./Bernard Baertschi (Hgg.), Die Philosophie und die Frage nach dem Glück/La philosophie et la question du bonheur, Studia philosophica 56 (1997), Bern/Stuttgart/Wien 1997, 125-138.

32 Ernst Bloch, Das Prinzip Hoffnung, Frankfurt am Main 1959, Bd. 3, 1628.

33 Augustinus, Confessiones X, 20. - Vgl. Paul Ricœur, der die Erinnerung an die ,unvordenkliche', unerinnerbare Vergangenheit (le passé immémorial) mit dem ,tiefen Vergessen zusammenbringt: La mémoire, l'histoire, l'oubli, 570-574.

${ }^{34}$ Maurice Merleau-Ponty, Phénoménologie de la perception, Paris 1945, 280; Jacques Derrida, „La différance“, in: ders., Marges de la philosophie, Paris 1972, 1-30, hier $21 \mathrm{f}$. 
die Artikulation einer Sehnsucht und eines Versprechens. Glückserinnerung in dieser emphatischen Gestalt ist nicht Erinnerung an eine erlebte Erfüllung, sondern an ein Versprechen.

Eine exemplarische Evokation findet dieses Glücksversprechen bei Adorno in der Erinnerung an Orte und Namen, wie er sie bei Proust geschildert sieht, aber auch, in den Odenwald verlegt, mit eigenen Kindheitserinnerungen assoziiert und ausdrücklich mit dem Zuhausesein, der Chiffre der Heimat verknüpft. ${ }^{35}$ Ähnlich erkennt er in einem Gedicht von Eduard Mörike („Auf einer Wanderung") „das Bild jenes Glücksversprechens, wie es noch heute am rechten Tag von der süddeutschen Kleinstadt dem Gast gewährt wird“, „das Gefühl der Wärme und Geborgenheit", das in Mörikes Gedicht aber kein „Glück im Winkel“ meint, sondern „die Utopie der nächsten Nähe“ mit jener „der äußersten Ferne“ verknüpft. ${ }^{36}$ So lautet auch Adornos Antwort auf die Frage nach dem Grund seiner Rückkehr: „Ich wollte einfach dorthin zurück, wo ich meine Kindheit hatte, am Ende aus dem Gefühl, dass, was man im Leben realisiert, wenig anderes ist als der Versuch, die Kindheit verwandelnd einzuholen. ${ }^{\text {"37 Indessen }}$ sind es Glückserinnerungen, die zuletzt nicht an ein Faktum, sondern an ein Versprechen erinnern und deren Gegenstand, wie das Bild der Versöhnung, der Anschauung entzogen bleibt, wie Adornos Beschreibung anschaulich festhält: Es ist eine Ahnung des Glücks, wie es „Namen von Dörfern verheißen wie Otterbach, Watterbach, Reuenthal, Monbrunn. Man glaubt, wenn man hingeht, so wäre man in dem Erfüllten, als ob es wäre. Ist man wirklich dort, so weicht das Versprochene zurück wie der Regenbogen. “" ${ }^{38}$

Im Wachhalten, Erwecken eines Nichtrealisierten berührt sich die Glückserinnerung mit dem Leidensgedächtnis, in dessen Schatten Geschichte nach Ricœur als Friedhof unerfüllter Hoffnungen und nicht gehaltener Versprechen

35 Vgl Adornos Brief an seine Mutter nach der Rückkehr aus dem Exil anlässlich eines Urlaubs in Amorbach: „Es ist schließlich doch das einzige Stückchen Heimat, das mir blieb“: Theodor W. Adorno, Briefe und Briefwechsel, Bd. 5, hg. von Christoph Gödde/Henri Lonitz, Frankfurt am Main 2003, 535 f. (Brief vom 24. September 1950).

36 Theodor W. Adorno, „Rede über Lyrik und Gesellschaft“, GS 11, 48-68, hier 61.

37 Theodor W. Adorno, „Auf die Frage: Warum sind Sie zurückgekehrt?“, GS 20.1, 394 f., hier 395. - Alexander Kluge meint, dass Adorno „Glück in der Kinderzeit kennengelernt hat oder meint, es kennengelernt zu haben“ und sich das „nie wieder ausreden“ ließ. Dabei geht es nicht um ein initiales Paradies, sondern ein aufscheinendes Glück, das gleich darauf vermisst wird; darunter leidet der Mensch, „und das macht ihm ein Gedächtnis, und nur wer dieses Gedächtnis hat, hat die Sehnsucht nach dem Glück in sich", nach einem Glück, von dem er eine Ahnung hat, die er nicht haben könnte, wenn er „nicht irgendeine Spur davon kennengelernt hätte“: „Es gibt kein richtiges Leben im falschen“. Theodor W. Adorno. Philosoph, Soziologe und Kritiker, Film von Henning Burk und Martin Lüdke, Hessischer Rundfunk/Westdeutscher Rundfunks 1989, https://www.youtube.com/watch?v=OMrtcGBFdMA (30. Oktober 2017).

38 Adorno, Negative Dialektik, 366. 
erscheint. ${ }^{39}$ Wenn Glückserinnerung diese Leerstelle akzentuiert und ins Affirmative wendet, so tut sie dies zwar nicht im Sinne der Bezugnahme auf eine bestimmte, temporal verortbare Erfüllung. Diese bleibt im Modus des Versprechens je aufgeschoben, nicht in ihrer Identität zu ver-gegenwärtigen, ähnlich wie der zurückliegende Grund dem Rückblick seit je sich entzieht. Dennoch wohnt ihr als Erinnerung ein affirmatives, bestimmendes Moment inne, das nicht im Potential der offenen Sehnsucht aufgeht, sondern gleichermaßen eine kognitive Setzung artikuliert. Es lässt sich daran festmachen, dass Erinnerung ihrem Wesen nach erkennend, wieder-erkennend ist. Paul Ricœur hat diesen Zug der Erinnerung nachdrücklich hervorgehoben und gezeigt, wie das ,kleine Wunder ${ }^{40}$ der Erinnerung - dasjenige, um das es dem Erinnern im Innersten geht und was seine Sehnsucht, sein Glück ausmacht - in der Wiedererkenntnis liegt, im Erlebnis, ein Gesuchtes zu finden, es wieder zu finden - auch wenn man es noch gar nie hatte und die Suche im Unbestimmten blieb. Erinnerung hat eine Erkenntniskraft, die als Wiedererkenntnis tiefer ist und weiter geht als die identifizierende Bezugnahme. Es ist dies in gewisser Weise eine Spiegelung der Wesensverwandtschaft, welche Platons Anamnesislehre herausstellt, wenn sie das Erkennen als ein Erinnern definiert, und die hier im Erinnern den genuinen Akt des (Wieder)Erkennens betont. Die gelingende, glückende Erinnerung, wie sie Ricœur unter dem Titel einer mémoire heureuse als „Leitstern“ des Gedächtnisses thematisiert, meint nicht die integrale Reproduktion früherer Ereignisse. Sie meint das lebendige Gewahrwerden, das Gegenwärtigwerden des Vergangenen im Jetzt. Es ist, verwandt dem ,dialektischen Bild' Benjamins, das Aufblitzen eines Erkennens, welches die Verhüllung der entzogenen Vergangenheit durchbricht und wie das Sagen des Nichtsagbaren anklingen lässt.

\section{Vorschein und vergebliches Warten}

In eigenartiger Korrespondenz zeigen sich die entgegengesetzten Pole der Erinnerung als Instanzen der Gegenwart eines Abwesenden, der Darstellung eines Nicht-Darstellbaren. Der Umgang mit den Grenzen der Sprache, der der menschlichen Lebensform unhintergehbar ist, nimmt vor ihrem Hintergrund ein mehrfaches Profil an. Es ist ein Umgang mit äußeren Grenzen, die durch den Inhalt des Nichtseinsollenden, Nicht-zu-Sagenden gesetzt sind, zugleich ein Umgang mit den inneren Grenzen einer verstümmelten Sprache, der konstitu-

39 Paul Ricœur, „Quel éthos noveau pour l’Europe?“, in: Peter Koslowski (Hg.), Imaginer l'Europe, Paris 1992, 109-116, hier 112; Burckhard Liebsch, „Die (gebrochenen) Versprechen der Moderne und die Zukunft der Geschichte. Zur Geschichtsphilosophie Ricœurs - mit Blick auf Kant, Levinas und Derrida", in: Allgemeine Zeitschrift für Philosophie 38/3 (2013), 299-320, hier 302.

40 Paul Ricœur, La mémoire, l'histoire, l'oubli, 556. 
tiven Sprachbehinderung des beschädigten Lebens und nicht-beredten Leidens, schließlich ein eigenes Sprechen unter Bedingungen der Sprachzerstörung, als transformierende Mimesis an das Verstummte oder als Versuch, die Spuren des Lebens zu erwecken und, wie Celans Dichtung, Adornos Lyrik-Verbot zu widerlegen. Doch nicht nur wie Lyrik, sondern wie Philosophie nach Auschwitz möglich sei, ist die drängende Frage für Adorno. Georg Picht hat die Herausforderung auf den allgemeineren Begriff eines Philosophierens ",nach Auschwitz und Hiroshima" gebracht: ein Titel, der nach Michael Theunissen darauf zielt, der Philosophie als „allumfassende Aufgabe“ die zuzuweisen, das „Hier und Jetzt" zu begreifen und in Auseinandersetzung mit der Abgründigkeit des Negativen „das Undenkbare“ $z u$ denken. ${ }^{41}$

Wenn das Ausloten der Grenzen von Vorstellung und Sprache besonderes drastisch in der Auseinandersetzung mit Leiden und Gewalt zum Tragen gekommen ist, so ist es nicht darauf beschränkt, sondern tangiert in anderer Weise auch die Glückserinnerung. Dabei stehen Leidens- und Glückserinnerung nicht nur nebeneinander als analoge Formen des Darstellungsentzugs, sondern nehmen wechselseitig aufeinander Bezug. Ihre Verweisung verkörpert die Verfassung negativistischen Denkens, das Negativität nicht in sich verschließen, sondern auf ihr Anderes hin öffnen, im Negativen die „,ersprengte Spur" des Anderen entziffern will. Es ist eine Spur, die aus der Tiefe der Erinnerung kommt: „Stets stammt sie aus dem Vergangenen, Hoffnung aus ihrem Widerspiel, dem, was hinab musste oder verurteilt ist. " ${ }^{42}$ In engster Weise sind Leidensgedenken und der Ausblick auf das Glück ineinander verschränkt, so dass auch das Glücksversprechen im Inneren des Leidensgedenkens aufbricht, wie Benjamins Engel der Geschichte den Sog der Zukunft mit dem Rückblick vereint. ${ }^{43}$ Zuletzt verbleibt ein Oszillieren, ob das Gedenken des Leidens aus sich heraus aufgebrochen und auf sein Anderes hin überschritten wird oder ob das Andere von außen und aus der Zukunft in die Erinnerung und das Leben der Menschen einbricht. Michael Theunissen hat in seiner Pindar-Lektüre in verwandtem Sinne die (vom Menschen geleistete), übersteigende` von der (aus dem Göttlichen kommenden)

${ }^{41}$ Georg Picht, Hier und Jetzt. Philosophieren nach Auschwitz und Hiroshima, 2 Bde., Stuttgart 1981; Michael Theunissen, „Die Einheit im Denken Georg Pichts. Zu seinem Buch ,Hier und Jetzt"“, in: Christian Link (Hg.), Die Erfahrung der Zeit. Gedenkschrift für Georg Picht, Stuttgart 1984, 356-368, hier 357.

42 Adorno, Negative Dialektik, 370.

43 Ähnlich beteuert Benjamin, dass „auf keinem Wege Neues zu hoffen“ sei „als auf dem der Heimkehr": Walter Benjamin, Aufzeichnungen 1933-1939, in: ders., Gesammelte Schriften, Bd. VI., 523. - Im Nachruf charakterisiert Adorno Benjamins Blick auf die Dinge als einen, „der aus der Perspektive des Toten die Welt sah, als läge sie in Sonnenfinsternis vor ihm: so wie sie dem Blick des Erlösten erscheinen mag“, als einen Blick, der im erkalteten Leben „Wärme und Hoffnung“ schenkt: Theodor W. Adorno, „Walter Benjamin. Zu Benjamins Gedächtnis“, GS 20.1, 169 f., hier 170. 
,einbrechenden 'Transzendenz unterschieden. ${ }^{44}$ Vielleicht gehört es zum Denken an der Grenze, wie es Adorno praktiziert, auch in dieser Frage nicht thetisch die eine gegen die andere Option auszuspielen.

Bei alledem ist bemerkenswert, wie sehr Adorno, bei allem Beharren auf der Notwendigkeit, das Nicht-Sagbare zu sagen, jede affirmative Setzung zurücknimmt und den Ausgriff auf das Andere im Vorläufigen, gleichsam in der Schwebe hält. Eine ausdrucksstarke Figur, in welcher diese Zurücknahme zum Ausdruck kommt, ist die "Situation des vergeblichen Wartens", wie Adorno sie exemplarisch in bestimmten Passagen bei Alban Berg realisiert sieht und als die Gestalt wahrnimmt, in der wohl „für uns metaphysische Erfahrung am stärksten ist. ${ }^{" 45}$ Das vergebliche Warten zeigt jenes an, das erwartet, vielleicht versprochen ist, dessen Eintreten aber ungesichert und fraglich bleibt. Gleichzeitig aber wird die Vergeblichkeit darin als eine erfahren, die womöglich nicht nur Vergeblichkeit ist - wie Adorno sie in jener „ungeheuren Stelle“ bei Proust beschrieben sieht, wo in der Schilderung des Todes von Bergotte wie eine Ahnung durchscheint, dass dieses Leben „kein ganz vergebliches sei“ “46. Im unauflösbaren Ineinander von Anspruch und Versagung, Vorschein und Warten bleibt negative Dialektik in Distanz zur affirmativen Metaphysik und deren negativer Unterlaufung gleichermaßen. Die Beharrlichkeit des Begehens dieser Grenze ist eine Weise, der Forderung, zu sagen, was nicht sich sagen lässt, zu antworten.

${ }^{44}$ Michael Theunissen, Pindar. Menschenlos und Wende der Zeit, München 2000, 217238 und passim.

45 Adorno, Metaphysik, 224; ders., Negative Dialektik, 368.

46 Adorno, Metaphysik, 211; ders., Negative Dialektik, 371. 